\title{
Syntactic- and morphology-based text augmentation framework for Arabic sentiment analysis
}

\author{
Rehab Duwairi ${ }^{\text {Corresp., }}{ }^{,}$Ftoon Abushaqra ${ }^{2}$ \\ 1 Department of Computer Information Systems, Jordan University of Science and Technology, Irbid 22110, Jordan \\ 2 Department of Computer Science, Jordan University of Science and Technology, Irbid 22110, Jordan \\ Corresponding Author: Rehab Duwairi \\ Email address: rehab@just.edu.jo
}

Arabic language is a challenging language for automatic processing. This is due to several intrinsic reasons such as Arabic multi-dialects, ambiguous syntax, syntactical flexibility, and diacritics. Machine learning and deep learning frameworks require big datasets for training to ensure accurate predictions. This leads to another challenge faced by researches using Arabic text; as Arabic textual datasets of high quality are still scarce. In this paper, an intelligent framework for expanding or augmenting Arabic sentences is presented. The sentences were initially labelled by human annotators for sentiment analysis. The novel approach presented in this work relies on the rich morphology of Arabic, synonymy lists, syntactical or grammatical rules, and negation rules to generate new sentences from the seed sentences with their proper labels. Most augmentation techniques target image or video data. This study is the first work to target text augmentation for Arabic language. Using this framework, we were able to increase the size of the initial seed datasets by 10 folds. Experiments that assess the impact of this augmentation on sentiment analysis showed a $42 \%$ average increase in accuracy, due to the reliability and the high quality of the rules used to build this framework. 
2 Syntactic- and morphology-based text augmentation 3 framework for Arabic sentiment analysis

5 Rehab M. Duwairi ${ }^{1}$, Ftoon M. Abushaqra ${ }^{2}$

$6{ }^{1}$ Department of Computer Information Systems, Jordan University of Science and Technology, Irbid

7 22110, Jordan.

$8{ }^{2}$ Department of Computer Science, Jordan University of Science and Technology, Irbid 22110, Jordan.

Corresponding Author:

12 P.O. Box 3030, Irbid, Irbid, 22110, Jordan.

13 Email address: rehab@,just.edu.jo 


\section{Syntactic- and morphology-based text augmentation 32 framework for Arabic sentiment analysis}

33

34

35

36

37

38

39

40

41

42

43

44

45

46

47

48

49

50

51

52

53

54

55

56

57

58

59

60

61

62

63

64

65

66

67

68

69

Rehab Duwairi ${ }^{1}$, Ftoon Abushaqra ${ }^{2}$

${ }^{1}$ Department of Computer Information Systems, Jordan University of Science and Technology, Irbid 22110, Jordan.

${ }^{2}$ Department of Computer Science, Jordan University of Science and Technology, Irbid 22110, Jordan.

Corresponding Author:

Rehab Duwairi ${ }^{1}$

P.O. Box 3030, Irbid, Irbid, 22110, Jordan

Email address: Rehab@just.edu.jo

\section{Abstract}

Arabic language is a challenging language for automatic processing. This is due to several intrinsic reasons such as Arabic multi-dialects, ambiguous syntax, syntactical flexibility, and diacritics. Machine learning and deep learning frameworks require big datasets for training to ensure accurate predictions. This leads to another challenge faced by researches using Arabic text; as Arabic textual datasets of high quality are still scarce. In this paper, an intelligent framework for expanding or augmenting Arabic sentences is presented. The sentences were initially labelled by human annotators for sentiment analysis. The novel approach presented in this work relies on the rich morphology of Arabic, synonymy lists, syntactical or grammatical rules, and negation rules to generate new sentences from the seed sentences with their proper labels. Most augmentation techniques target image or video data. This study is the first work to target text augmentation for Arabic language. Using this framework, we were able to increase the size of the initial seed datasets by 10 folds. Experiments that assess the impact of this augmentation on sentiment analysis showed a $42 \%$ average increase in accuracy, due to the reliability and the high quality of the rules used to build this framework.

Keywords: Text Augmentation, Sentiment Analysis, Arabic Text, Natural Language Processing, Morphology-Based Augmentation.

\section{Introduction}

Arabic language is considered the most widely spoken language among the Semitic languages [1, 2]. It is also one of the popular languages in the world. As the statistical studies in 2019 mentioned [3], Arabic language is spoken by nearly 319 million people and is ranked the fifth 
70 between the world's languages after Chinese, Spanish, English, and Urdu Indian. Arabic native

71 speakers are distributed throughout the Arab World as well as many other nearby areas. Also,

72 Arabic has around 30 modern varieties or dialects; one of them is the standard form MSA

73 (Modern Standard Arabic) [4]. In 2012, the United Nations Economic and Social Commission

74 for West Asia (ESCWA) mentioned that Arabic language has achieved the highest growth rate

75 on the Internet compared to other languages. Therefore, recently digital Arabic content on the

76 internet became fairly large. However, this does not deny the reality that Arabic is considered a

77 highly ambiguous language, especially when trying to analyze, classify and process Arabic data

78 automatically.

79 Recently, many efforts have investigated the Arabic language whether to analyze the text [5, 6,

80 7], parse statements [8, 9], analyze sentiment [10, 11, 12, 13], recognize speech [14, 15],

81 translate statements [16, 17], or detect depression [18]; all these applications require the

82 existence of comprehensive Arabic datasets. Building a dataset is not an easy task, as it requires

83 tremendous effort, time and cost. Also, the recent application of machine learning and deep

84 learning requires huge datasets which contain billions of records. For example, training a

85 sentiment classifier using deep learning methods requires huge data properly labelled with

86

87

88

89

90

91

92

93

94

95 polarity information. Therefore, an automatic expansion for Arabic datasets is very favorable, especially when knowing that manually collecting and annotating data are troublesome [19].

Sentiment analysis is the task of processing data, mainly textual, in order to determine its polarity, i.e., positive, negative, or neutral $[20,21]$. This task has several real-world applications with great impact on important domains such as business [22], politics [23, 24], tourism [25] and marketing [26]. In general, sentiment analysis could be treated as an unsupervised learning task [27], supervised learning task [28], or a hybrid of both. Unsupervised learning for sentiment analysis relies on sentiment lexicons. By comparison, supervised learning requires the existence of annotated or labelled data to train the classifiers. Ideally, data labeling for sentiment analysis mandates that each instance must be assigned a label from: positive, negative, or neutral. This task of labelling is usually human-based and thus it is costly. Corpora for sentiment analysis are usually gathered from social media; and due to the multilingual nature of such media, several researchers directed their efforts towards multilingual sentiment analysis [29, 30, 31].

Deep learning has received unprecedented attention in recent years and provided state-of-the-art results in many fields including sentiment analysis [32, 33, 34, 35, 36]. However, deep neural networks need large amounts of data to train and tune their parameters. Data augmentation is a technique for expanding the datasets, and it was paired with deep learning applications. It has been used successfully with vision data and recently has received attention with textual data. Augmenting data that was initially labelled for sentiment analysis involves generating new sentences relying on the existing ones. The simplest form is to use the synonyms of the words to create new sentences with the same labels as the original ones. In this work, a framework for extending the size of datasets that were originally labeled for sentiment analysis is presented. 
110 Specifically, in focus on the Arabic syntax, grammar and morphology to create new sentences

111

112

113

114

115

116

117

118

119

120

121

122

123

124

125

126

127

128

129

130

131

132

133

134

135

136

137

138

139

140

141

142

143

144

145

146

147

148

149

with the same labels or opposite labels as explained in Section 4.

The syntax of Arabic language is complex [37, 38] - as several matching cases are possible between words in the same sentence, while in addition, each word has several synonyms.

Therefore, it is possible to generate tens of variants for an Arabic sentence while preserving its meaning. This task can be automated if the system is able to parse the statement and link it to lexical resources. Parsing is the process where each word in the text is labeled with its part of the speech tag (Verb, Object, Subject, etc.). However, parsing is not a simple process especially for Arabic language where the structure and order of the words are not specified. The Natural Language Processing Group at Stanford University has built an open-source parser [39]. Stanford Parser provides a set of natural language processing functions. Mainly, it was built for English, later on, many developers have carried out extensive work to improve the code and the grammatical rules to make it more comprehensive. As a result, this parser has been extended to include languages other than English, such as Chinese, German, Italian and Arabic. The parsing tool takes a text file as input and generates the base forms of words, normalizes and interprets dates, times, and numeric quantities. Finally, it analyzes the grammatical structure of the sentences. The output of the parsing process can be presented in several forms, such as phrase structures, trees, or dependencies.

For building the framework, initially the Stanford Arabic Parser was used to generate the parse trees of Arabic sentences. Afterwards, the augmentation rules generated were used on these trees, to generate several equivalent parse trees for the original sentences utilizing Arabic morphology, syntax, synonyms, and negation particles. These augmentation rules can be broadly divided into: (1) rules which alter or swap branches of the parse trees as per Arabic syntax and thus generate new sentences with the same labels (2) rules which generate new parse trees by utilizing the synonyms of words in these sentences, and also generate new sentences with the same original labels (3) rules which insert negation particles into the sentences and thus generate new sentences with opposite labels. It is worth mentioning here that the work in this paper addresses text augmentation for sentiment analysis. This means that the labels of the investigated sentences are either neutral, positive or negative. Applying the sets of rules described in (1) or (2) above will generate new sentences with the same labels as the input sentences. By comparison, applying the set of rules described in (3) as aforementioned, generates new sentences with opposite labels to the input sentences. Experiments proved the viability and effectiveness of the augmentation framework by running three experiments using three datasets. The size of the original datasets substantially increased and the generated sentences were of high quality.

The rest of this article is organized as follows: Section 2 briefly describes the related literature works. Section 3 explains the properties of Arabic language. Section 4 explains the design of the transformation rules which are the core of the augmentation framework. Section 5 describes the

Peer] Comput. Sci. reviewing PDF | (CS-2020:10:54813:1:2:NEW 27 Feb 2021) 
150

151

152

153

154

155

156

157

158

159

160

161

162

163

164

165

166

167

168

169

170

171

172

173

174

175

176

177

178

179

180

181

182

183

184

185

186

187

188

189

implementation of the framework. Section 6 demonstrates the experiments which were carried out to assess the effectiveness of the proposed work. And finally section 7 summarizes the conclusion of this work.

\section{Related Work}

This section describes related studies which have utilized Arabic WordNet as a component of frameworks. It also describes the related work which addresses data augmentation.

\section{Arabic WordNet}

WordNet [40] is a large linguistic database, or hierarchical dictionary, which was initially developed for the English language. It has been very useful for the fields of computational linguistics and Natural Language Processing [41]. Because of its structure, the WordNet differs from other standard dictionaries, where it groups words based on their meanings. The English WordNet lexicon [42] is divided into syntactic categories that contain (nouns, verbs, adjectives, and adverbs). It should be noted here that function words are deleted. However, WordNet grouped synonyms using the meaning (thesaurus) rather than the form (dictionaries). It also represents words redundantly - where a given word may appear in noun, verb and adverb syntactic categories. The WordNet consists of four parts [40]: (1) lexicographers source files; (2) the tool to convert these files into the lexical database; (3) the lexical database; (4) software tools that are used to access the database.

WordNet has been very useful as it was used to build many Natural Language Processing applications, Information Retrieval, term expansion and document representations [43]. For example, Varelas et al. [44] compared the performance of using single ontology and different ontologies for the semantic similarity methods. Single ontology experiments were performed using the WordNet and it showed better performance in the results.

However, many efforts have been reported to adapt WordNet for other languages, such as WordNets for European languages [45], and French and Slavonian WordNets [46]. By comparison, Arabic WordNet [47] used the same development approach for word representation of Princeton WordNet to keep it compatible with other Word-Nets' structures. Arabic WordNet is a lexical database for Modern Standard Arabic, with two main linguistic categories (verbs and nouns). First, the important concepts that represent the core WordNet were extracted, then specific concepts for the Arabic language were developed along with other concepts that were manually translated to the most convenient synset from other languages. It was developed using MySQL and XML [47]. Finally, the Arabic WordNet ended up with 11,270 synsets (2538 verbs, 7961 nominal, 110 adverbs, and 661 adjectives) with 23,496 Arabic expressions. Table 1 presents detailed information about the statistical properties of Arabic WordNet.

Several researchers have targeted extending Arabic WordNet. For example, in the work reported in [48, 49], the authors automatically extracted named entities from Arabic Wikipedia.

Subsequently, they attached these entities as instances to the synsets of Arabic WordNet and 
190

191

192

193

194

195

196

197

198

199

200

201

202

203

204

205

206

207

208

209

210

211

212

213

214

215

216

217

218

219

220

221

222

223

224

225

226

227

228

229

finally created a link to their counterparts in English WordNet. Moreover, Badaro et. al [50] introduced an automatic method for expanding Arabic WordNet - where they formulated the problem as a link prediction problem.

Shoaib et al. [51] used the relationships in Arabic WordNet in order to build a model for semantic search in the Holy Quran. The proposed model improved searching and retrieving of the related verses from the Holy Quran without mentioning a specific keyword in the query. The model works in two stages. Namely, it identifies one sense of the query word using Word Sense Disambiguation, then it extracts out all the synonyms of the identified sense of the word. AlMaayah et al. [52] have also worked on the Holy Quran, where the researchers have built a model that extracts the synonyms and builds the Quranic Arabic WordNet. This net was built based on the Boundary Annotated Quran Corpus, lexicon resources, and traditional Arabic dictionaries. The final model was able to link the Holy Quran words that have the same meaning and generate sets of synsets using the vector space model. The Quranic Arabic WordNet has 6918 synsets from 8400 unique word senses. In other studies, the researches have tried to extract semantic relationships between words, and provide models to represent ontological relations for the Arabic content on the internet. These representations are useful to facilitate the analyses and processing of Arabic text. Al Zamil and Al-Radaideh [53] have used the semantic features that were extracted from the text along with syntactic patterns of relationships to provide models that are able to automate the process of ontological relations extraction. The extracted features are used to construct generalized rules which were used to build a classifier. The classifier presents each concept with its designated relationship label.

\section{Data Augmentation}

Data augmentation is a technique that is used to increase the size of datasets and preserve the labels at the same time. It became popular with deep learning networks as they require training on huge datasets to secure high accuracies $[54,55,56,57]$. Extending the size (number of samples) in a dataset, especially for under-represented classes, is mainly depended on generating perturbed replicas of the class samples. This technique has proved its success in image classification such as the work reported in [54, 58, 59]; 3D pose estimation as reported in [60]; speaker language identification as described in [61]; recognition of audio-visual effect [62]; and the classification of the environmental sound [63].

On the other hand, data augmentation is limited when dealing with textual data. This is due to the very difficult definition and standardization of specific rules or transformations that preserve the meaning of the produced textual data [19]. Basically, the main approach that works to increase the size of textual data, and preserves text meaning, is to use the synonyms of words, relying on lexical resources such as WordNet.

The works reported in $[64,65]$ have used a synonyms-based approach for augmenting textual data. As the synonyms are very limited, the proposed sentences are not very different and 
230

231

232

233

234

235

236

237

238

239

240

241

242

243

244

245

246

247

248

249

250

251

252

253

254

255

256

257

258

259

260

261

262

263

264

265

266

267

268

269

numerous from the original texts. Therefore, Kobayashi [19] has proposed the contextual augmentation method, which is a state-of-the-art method to augment words, and produce more varied sentences. The author used words predicted by the bidirectional language model (LM) instead of using synonyms. The proposed approach was able to present a wide range of substitute words and it has been tested with two classifiers using recurrent or convolutional neural networks where it improves the overall performance. Georgios Rizos et al. [66] targeted extending a text used for hate speech detection relying on synonyms lists, wrapping the word token around the padded sequence, and finally applying class-based conditional recurrent neural language generation. The authors state that they achieved a 5.7\% increase on Macro-F1 and a $30 \%$ in recall when extending the datasets using their three text extensions methods.

The work reported in [67] has described a framework for augmenting tweets based on ConceptNet and Wikidata. The authors suggested two methods for improving the quality of tweets by first appending terms extracted from ConceptNet and Wikidata to the existing tweets but not increasing their numbers. Secondly, they generated new tweets by replacing words or terms in the original tweets with terms extracted from ConceptNet and Wikidata. This approach is close to the approaches which utilize synonyms.

In a similar study, Kolomiyets et al. [68] replaced the headwords with a substitute word predicted from the Latent Words in the language model. The authors only used the top k score words as a substitute. Mueller and Thyagarajan [69] substituted random words in sentences with their synonyms to generate new sentences. Subsequently, they trained a siamese recurrent network to compute the similarity between sentences. Wang and Yang [65] employed word embedding to increase the size of the training data. Specifically, they replaced a given word with its nearest neighbor word vector.

As it can be seen from the above literature, most of the existing augmentation techniques address image or audio data and less work addresses text augmentation. In this regard, it should be mentioned that no work addresses Arabic text augmentation. The current proposed framework is substantially different from text augmentation which relies on the replacement of words by their synonyms. On the other hand, it utilizes the rich syntax and grammar of the Arabic language in order to generate transformation rules, that are subsequently used to generate new sentences based on seed sentences.

\section{Arabic Language Properties}

Arabic language is one of the Semitic languages. It consists of twenty-eight basic letters. Several Arabic letters change their shapes based on their location in the word. For example, the letter (w) has the shape (- when it is located at the beginning of the word, the shape (- when it is located at the middle of the word, (w) when it is located at the end of the word but connected to the previous letter, and (w) when it comes at the end of the word but disconnected from the previous letter. Arabic is an inflectional language that is written from right to left. The following three subsections provide background about Arabic language. 
270

271

272

273

274

275

276

277

278

279

280

281

282

283

284

285

286

287

288

289

290

291

292

293

294

295

296

297

298

299

300

301

302

303

304

305

306

307

308

\section{Arabic Morphology}

Morphology is the structure of words. The morphology of Arabic language is complex but systematic - where there are two ways to build a word in Arabic: derivation and agglutination. ف، ( The derivation is a way of generating stems from a list of roots; based on three basic letters " (ع) ل ل can generate the following stems:
- Study "darasa نَرَ" دَ"
- Scholar "dAris "درس"

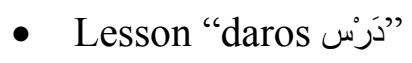
- Teacher "mudaris مُعَرس"
- Schools "mdAris مدارس"

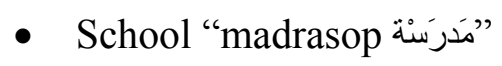

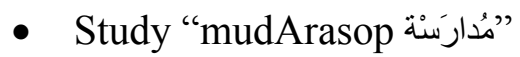

The second way to build words in Arabic language is agglutination. In this way, the words are built by adding affixes to the word. These affixes could be prefixes at the beginning of words such as (است، تم ، تن، (ن) (ان) infixes in the middle of the word (such as I), or suffixes at the end of the

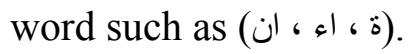

\section{Arabic Syntax}

In Arabic scripts, the sentence has two types or categories (nominal and verbal). Each type has its own grammar and rules. The nominal sentence, in Arabic, consists of a subject (Almubtada) and predicate (Alkhabar). The normal order is that the subject is followed by the predicate but in

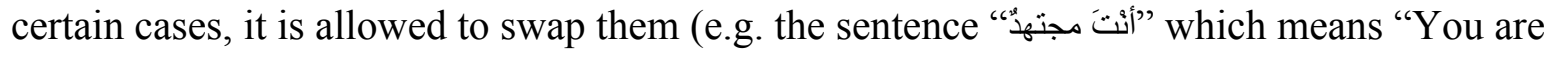
diligent" could be "مجتهد" أنتن"). The subject in the nominal sentence can be Noun, Pronoun or Number while the predicate can be Singular Noun, Adverb, Preposition, Nominal sentence, or Verbal Sentence.

The verbal sentence in Arabic, like in many other languages, consists of Verb (V), Subject (S), and Object $(\mathrm{O})$ without a specific order, which means that the order of verbal sentences could be: VSO, VOS, SVO or VOS. Additionally, in Arabic language diacritics, prefixes and suffixes are used to represent gender. Therefore, the absence of diacritics can create ambiguity and might change the meaning.

\section{Diacritics}

One of the Arabic language features is the diacritics that are written above or underneath its letters. Diacritics are small vowel marks that represent three short vowels (a, i, u). They are used to regulate and control the letters and pronunciation. Therefore, diacritics have a huge effect on the text and its meaning, removing them may lead to morphological-lexical and morphologicalsyntactical ambiguities. For example, the word (nEm) (نعم) has the meaning 'Yes' if it was written 
309

310

311

312

313

314

315

316

317

318

319

320

321

322

323

324

325

326

327

328

329

330

331

332

333

334

335

336

337

338

339

340

341

342

343

344

345

346

347

348

349

350

351

(naEom نَ), while it means 'graces' if it was written (niEm The basic diacritics of Arabic language are:

- Fatha: symbolized as an italic score on the top of the letter (- ).

- Dma: symbolized as a small (و) letter on the top of the letter (- ).

- Ksra: symbolized as an italic underscore on the bottom of the letter (- ).

- Sokon: symbolized as a small circle on the top of the letter ( ).

\section{Transformation rules definition}

As a first step, clear definitions of Arabic grammar rules were specified. These rules include specifications for nominal sentences, verbal sentences, questions, verbs, adjectives, pronouns, prepositions, conjunctions, and numbers. These defined grammar-based rules were represented using the Stanford Arabic parser tagset. Table 2 lists these tags in full details.

Table 3, on the other hand, summarizes the core concepts of this research - it depicts, in the second column, grammar rules for valid sentences in Arabic. The third column of Table 3 lists equivalent grammar rules which were derived from the original rules listed in the second column. The importance of these rules is that sentences that respect the grammar rules listed in the second column could be mapped to new sentences which fulfill the grammar rules listed in column 3, and still have the same label for the classifiers. The following statements show example sentences from Arabic which respect grammar rules in Table 3 and show how these sentences are transformed into new sentences, $\rightarrow$ means that the RHS of the rules are equivalent to the LHS:

RULE 1: DTNN +ADJ $\rightarrow$ ADJ +DTNN

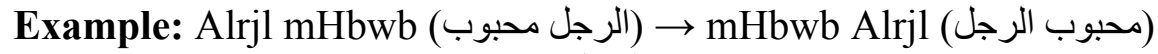

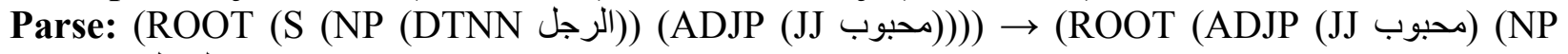
(الرجل)(DTNN) (الر (DT)

RULE 2: $\mathrm{NN}+\mathrm{ADJ} \rightarrow \mathrm{ADJ}+\mathrm{NN}$

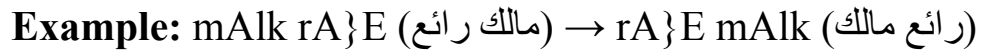

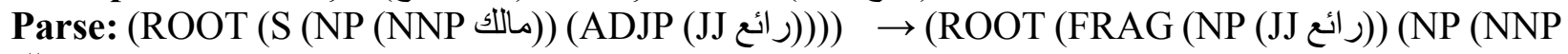
(مالك)(R))

RULE 3: $\mathrm{DTNN}+\mathrm{NN} \rightarrow \mathrm{NN}+\mathrm{DTNN}$

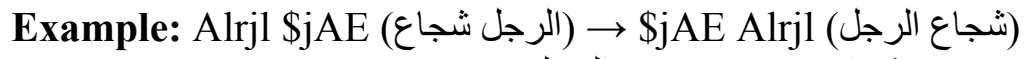

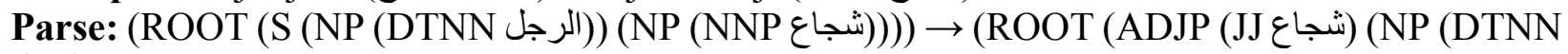

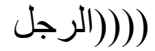

RULE 4: $\mathrm{NN}+\mathrm{NN} \rightarrow \mathrm{NN}+\mathrm{NN}($ swap)

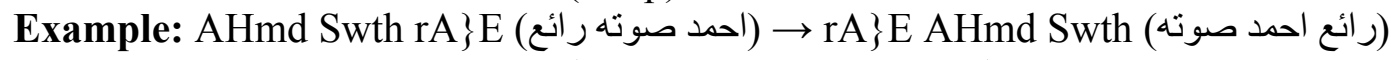

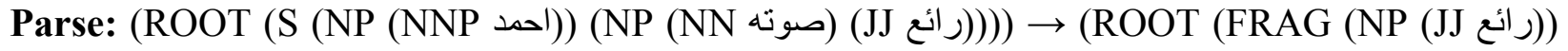

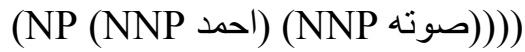

RULE 5: $\mathrm{NN}+\mathrm{DTNN} \rightarrow \mathrm{NN}+\mathrm{DTNN}$ 


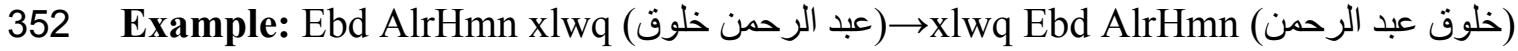

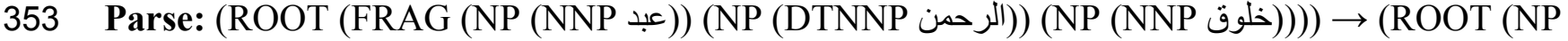

354

355

356

357

358

359

360

361

362

363

364

365

366

367

368

369

370

371

372

373

374

375

376

377

378

379

380

381

382

383

384

385

386

387

388

389

390

391

392

393

394

395

396

397

RULE 6: DTNN +DTNN $\rightarrow$ DTNN + DTNN(swap)

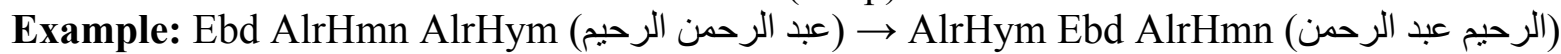

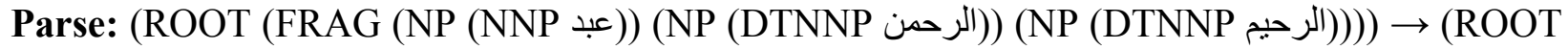

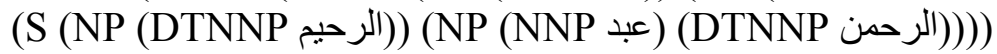

RULE 7: ADJ +ADJ $\rightarrow$ DTNN + DTNN (sawap)

Example: AlftAp Aljmylp mjthdp (الفتاة الجميلة مجتهدة) AlftAp mjthdp Aljmylp (الفتاة مجتهدة الجميلة)

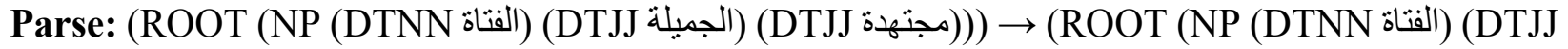
(مجتهدة (الجميلة (DTJ)) (مجن)

RULE 8: $\mathrm{PP}+(\mathrm{NN}+\mathrm{DTNN}) \rightarrow$ place them in the beginning and reverse the sentence

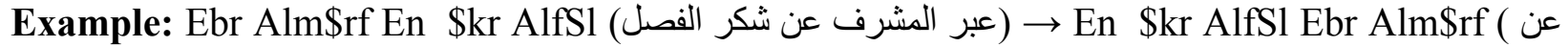
(شكر الفصل عبر المشرف (Rx)

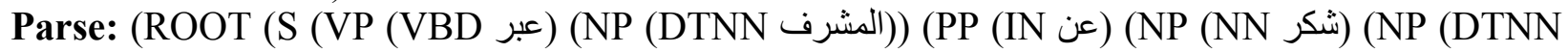

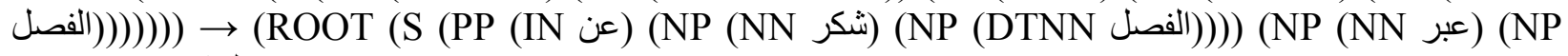

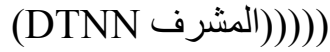

RULE 9: PP + DTNN $\rightarrow$ place them in the beginning and revese the sentences

Example: bAsm yqdm \$y\}A mn AlfkAhAt (باسم يقدم شيئً من الفكاهات) mn AlfkAhAt bAsm yqdm \$y\}A (من الفكاهات باسم يقدم شيئ)

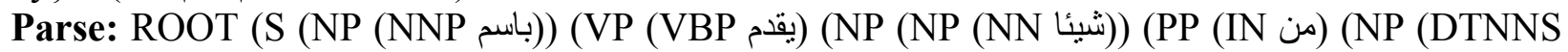

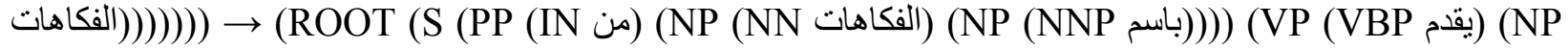
$(\mathrm{NN}($ (نيئl)()))

RULE 10: $P P+($ Special character VB $\mid N N) \rightarrow$ place them in the beginnig and reverse the sentence

Example: tSAdq mE Al*\}Ab ElY >n ykwn f>sk mstEdA (تصادق مع الذئاب على أن يكون فأسك مستعد)

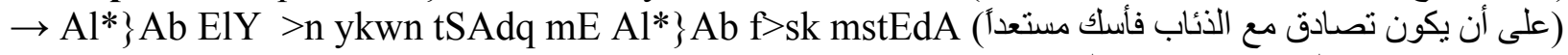

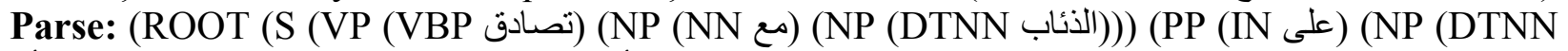

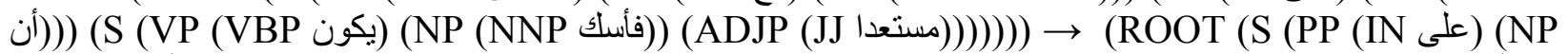

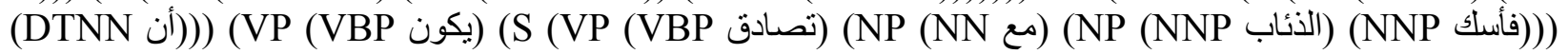
$(\mathrm{ADJP}(\mathrm{JJ}($ مستعد))))))

RULE 11: Wh-prounoun at the end of the sentences $\rightarrow$ Move it to the beginning

Example: njH Al*y *hb AlY Almdrsp (نجح الذي ذهب الى المدرسة) Al*y *hb AlY Almdrsp njH

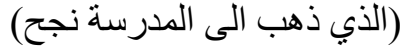

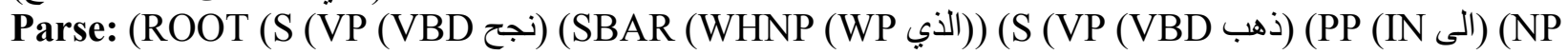

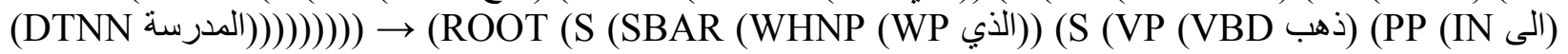

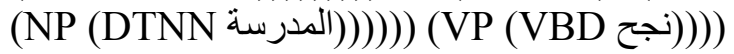

RULE 12: Special adverb $+(\mathrm{NN}|\mathrm{VB}|($ special character VB $\mid \mathrm{NN})) \rightarrow(\mathrm{NN}|\mathrm{VB}|$ (special character $\mathrm{VB} \mid \mathrm{NN}))+$ Special adverb 


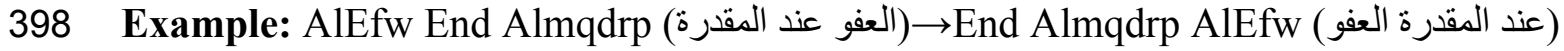

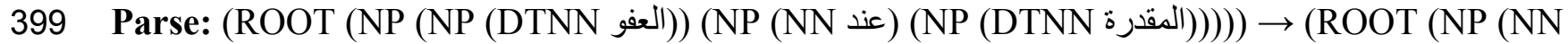

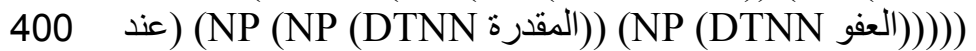

401 Example: frH Alwld bxbr AlrHlp qbl>n y*hb (فرح الولد بخبر الرحلة قبل أن يذهن qbl >n y*hb frH

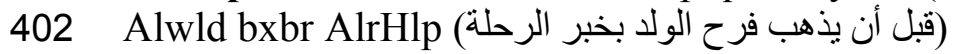

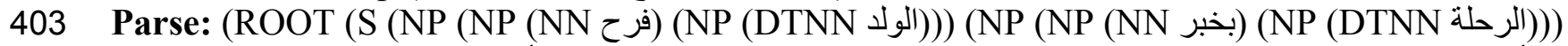

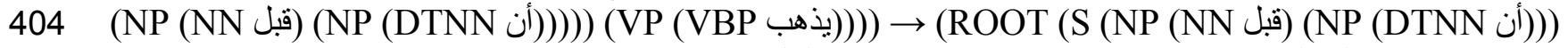

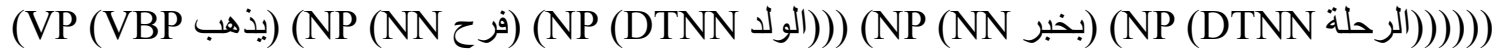

RULE 13: Pronoun + (NN |VB | ADJ) $\rightarrow(\mathrm{NN}|\mathrm{VB}| \mathrm{ADJ})+$ Pronoun

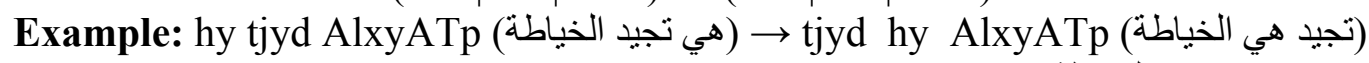

410

411

412

413

414

415

416

417

418

419

420

421

422

423

424

425

426

427

428

429

430

431

432

433

434

435

436

437

438

439

440

441

442

443

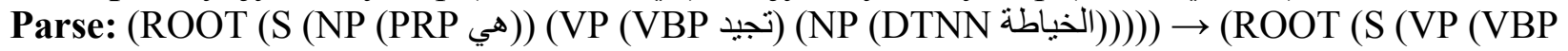

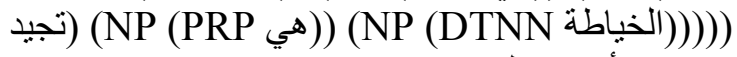

Example: Ant rjl krym (ري (رجن رجل انت كريم) rjl krym Ant)

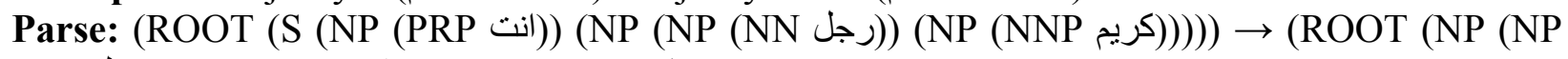

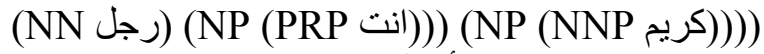

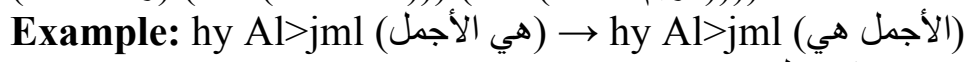

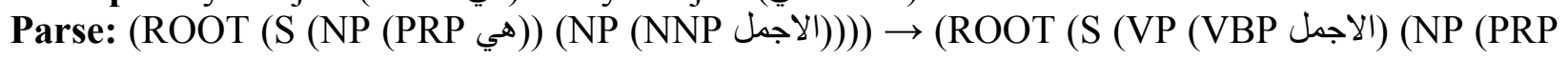
هي (ه))))

RULE 14: $(\mathrm{NN} \mid \mathrm{DTNN})+\mathrm{VB} \rightarrow \mathrm{VB}+(\mathrm{NN} \mid \mathrm{DTNN})$

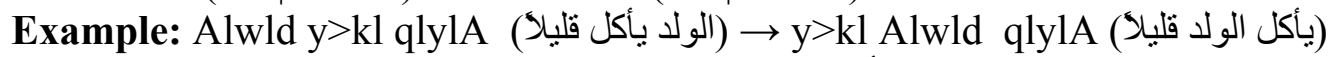

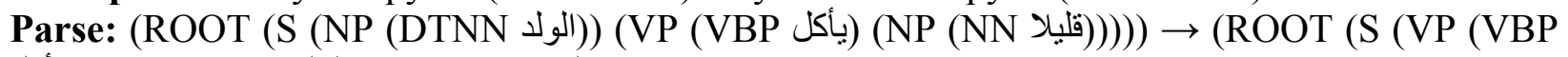

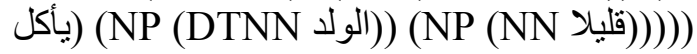

Example: bAsm yqdm \$y\} A mn AlfkAhAt (باستم يقدم شيئا من الفكاهات) $\rightarrow$ yqdm bAsm \$y\} A mn AlfkAhAt (بقدم باسم شيئا من الفكاهات)

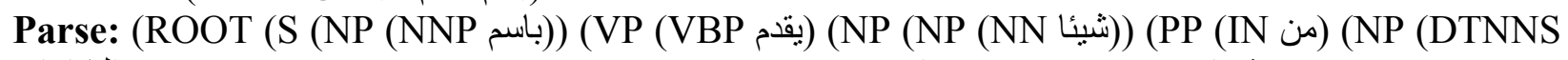

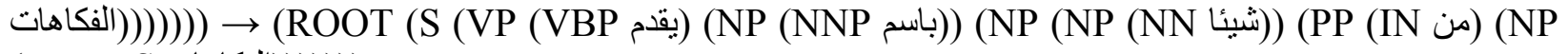
(DTNNS (الفكاهات)()))))

RULE 15: NN+ (Special-character $+\mathrm{VB}) \rightarrow($ special-character $+\mathrm{vb})+\mathrm{NN}$

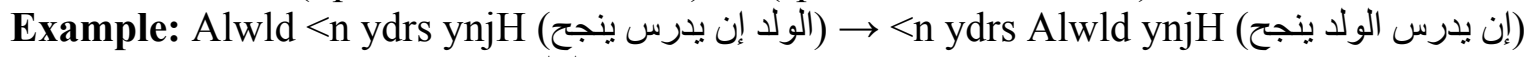

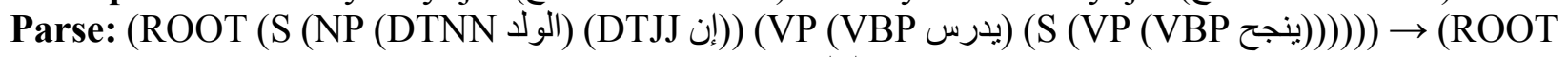

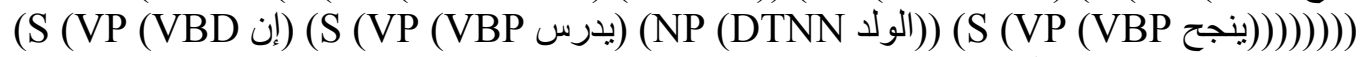

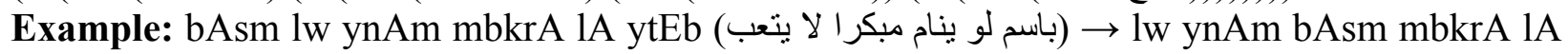

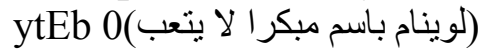

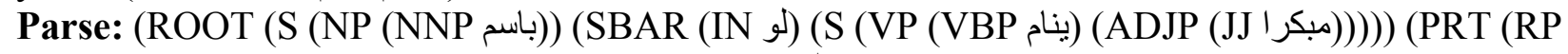

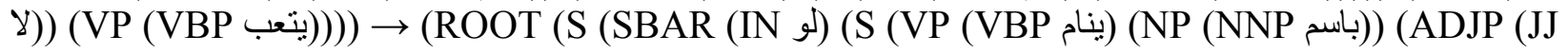

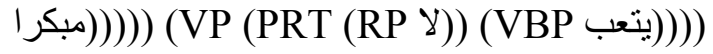

RULE 16: VB+(NN|DTNN) $\rightarrow(\mathrm{NN}+\mathrm{DTNN})+\mathrm{VB}$

Example: wqE Alwld ElY Al>rD (وقع الولد على الأرض) Alwld wqE ElY Al>rD الولد وقع على ) الوضى) (الأرض

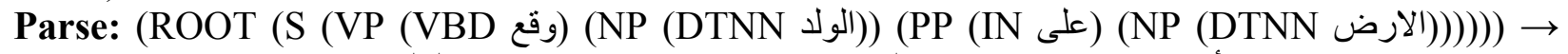

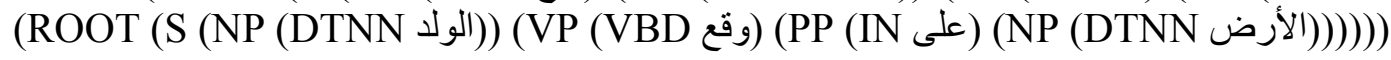


444 RULE 17: VB+(Special-character $+(\mathrm{NN} \mid \mathrm{DTNN})) \rightarrow$ Special-character $+(\mathrm{NN} \mid \mathrm{DTNN}))+\mathrm{VB}$

445 Example: Elmt >n AlwfA' Sfp EZymp (علمت أن الوفاء صفة عظيمة) > >n AlwfA' Elmt Sfp EZymp $446 \quad($ أن الوفاء علمت صفة عظيمة)

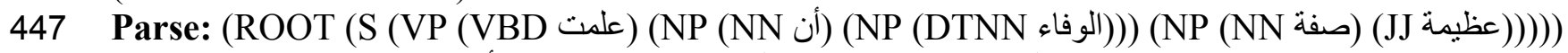
448 449

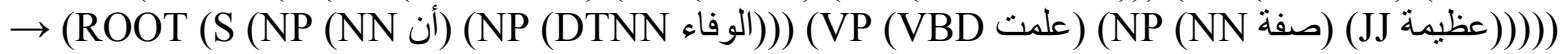

RULE 18: (Special-character $+\mathrm{VB})+($ Special-character $+(\mathrm{NN} \mid \mathrm{DTNN})) \rightarrow($ Special-character + $(\mathrm{NN} \mid \mathrm{DTNN}))+($ Special-character $+\mathrm{VB})$

Example: ln >Elm >n AlwfA' Sfp EZymp (لن أعلم أن الوفاء صفة عظيمة) >n AlwfA' ln >Elm Sfp EZymp (أن الوفاء لن أعلم صفة عظيمة)

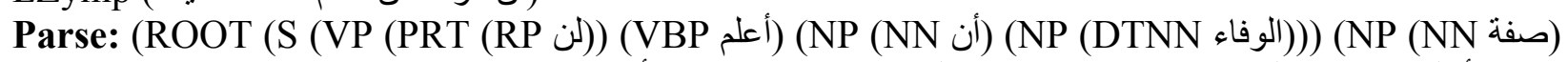

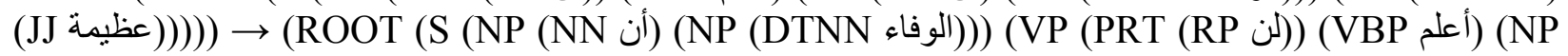

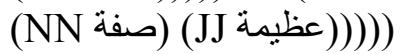

RULE 19: (Special-character +VB) +(NN|DTNN) $\rightarrow(\mathrm{NN} \mid \mathrm{DTNN})+($ Special-character +VB)

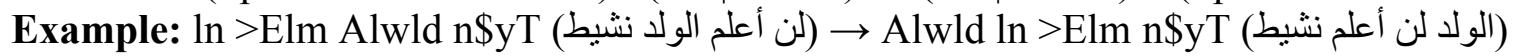

460

461

462

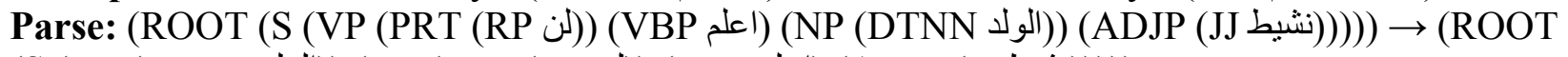

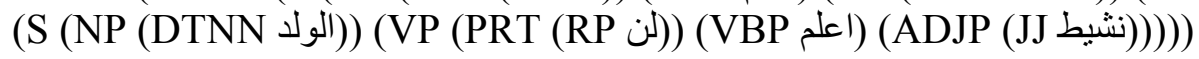

RULE 20: (Special-character $+(\mathrm{NN} \mid \mathrm{DTNN}))+\mathrm{VB} \rightarrow \mathrm{VB}+($ Special-character $+(\mathrm{NN} \mid \mathrm{DTNN}))$ Example: >n AlwfA' yEml mEk (ان الوفاء يعمل معك) $\rightarrow$ yEml >n AlwfA' mEk (يعمل أن الوفاء معك))

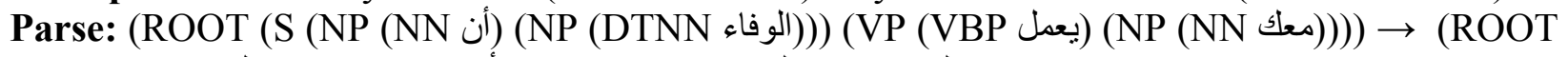

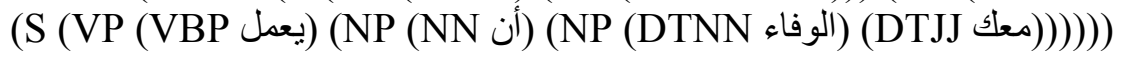

RULE 21: (Special-character $+(\mathrm{NN} \mid \mathrm{DTNN}))+($ Special-character $+\mathrm{VB}) \rightarrow($ Special-character +VB) +

470

471

472

473

474

475

476

477

478

479

480

481

482

483

484

485

486

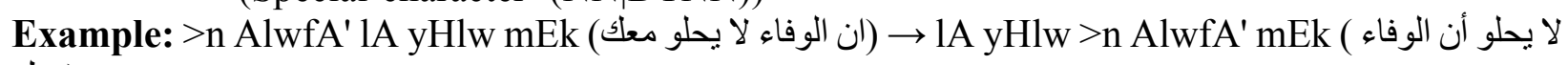
(معك)

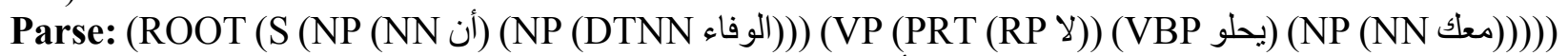

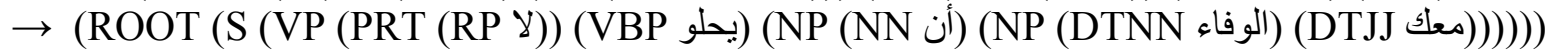

RULE 22: $\mathrm{CD}+(\mathrm{NN}|\mathrm{DTNN}| \mathrm{VB}) \rightarrow \mathrm{CD}+(\mathrm{NN}|\mathrm{DTNN}| \mathrm{VB})$

Example: njH TAlb fy Altwjyhy (نجح 15 طالب في النوجيهي) fy Altwjyhy njH TAlb (ني النوجي) (نجح 15 طالب

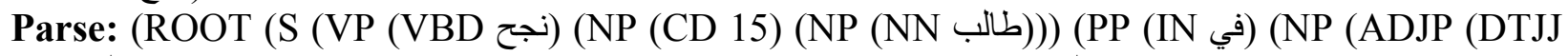

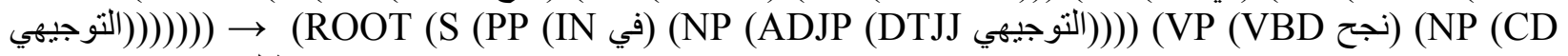
15) (NP (NN طالب (ط)))))

RULE 23: WH-Adverb $+(\mathrm{NN}|\mathrm{VB}| \mathrm{DTNN} \mid($ Special-character $+(\mathrm{NN} \mid \mathrm{DTNN})) \mid$ (Special-character $+\mathrm{VB})) \rightarrow(\mathrm{NN}|\mathrm{VB}| \mathrm{DTNN} \mid($ Special-character+(NN|DTNN $)) \mid($ Special-character $+\mathrm{VB}))+\mathrm{WH}-$ Adverb

Example: kyf kl AlnAs y>klwn (كيف كل الناس يأكلون) kl AlnAs kyf y>klwn (كل الناس كيف يأكلون) (كل)

Parse: (ROOT (SBARQ (WHADVP (WRB كيف) (S (NP (NOUN_QUANT كل) (كل) (S) (ST) (DTNN

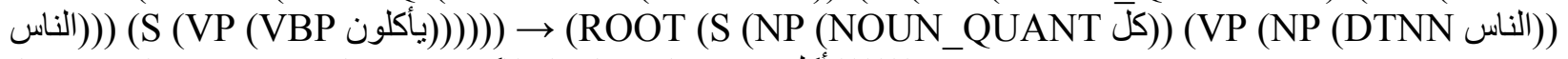

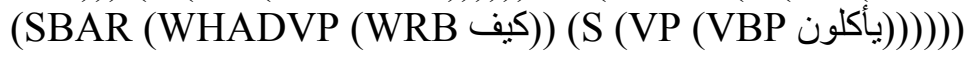


491 Extensive experiments, showed that the Arabic Stanford parsing is not very accurate especially 492 for the adverbs and negation words. This will adversely affect the system by generating wrong

493

494

495

496

\section{7}

498

499

500

501

502

503

504

505

506

507

508

509

510

511

512

513

514

515

516

517

518

519

520

521

522

523

524

525

526

527

528

529 synonyms for the sentences. Therefore, there existed the need for declaring our own list of some adverbs, negation and special words because Stanford Parser does not assign the proper labels, as expected. These lists are presented in Table 4.

\section{Description of Framework}

The general framework is illustrated in Figure 1. In the first step, a sentence with its label is passed to the system and it is converted to its canonical form (i.e. its parse tree). Secondly, multiple equivalent sentences (parse trees) are generated from the input sentence by replacing words with their synonyms. The synonyms are generated using Arabic WordNet. Thirdly, multiple variants of the sentences (parse trees), which were generated in step 2, are produced based on the transformation grammatical rules described in Table 3. The sentences generated in step 2 and step 3 all have the same label as the original input sentence. The Negation module is optionally called if we want to infuse negation particle into the generated sentences, and thus substantially increasing the number of generated sentences. The generated sentences from the Negation module have opposite labels to the input sentence and its variants. The following subsections describe, in detail, each developed module.

\section{Generate synonyms using Arabic WordNet}

The Arabic WordNet browser is free and publicly available. It uses a locally-stored database of Arabic data in XML format - where words of the same meaning are linked through pre-defined lexical relations. Furthermore, the interface is modeled on the European language WordNet interface; hence, it contains the basic components with additional Arabic components. However, the performance of the Arabic WordNet is not satisfactory when compared with other WordNets. For example, the Arabic WordNet contains only 9.7\% of the Arabic lexicon, while the English WordNet covers $67.5 \%$ of the English lexicon. Also, the Arabic WordNet synsets are linked only through hyponymy, synonymy, and equivalence; correspondingly seven semantic relations are used in the English WordNet. However, since the main goal is generating the synonyms of the words, the limitation of the Arabic WordNet did not substantially affect the work. Also, to avoid the noise caused by diacritics, only the first top five synsets in each synonyms list were considered. Table 5 shows the first eight synsets for the Arabic word "Man - رجل". As it can be seen from Table 5, the further we go deeper in generating synonyms, the higher the chance of generating wrong synonyms. The last two entries in Table 5 correspond to "leg and foot" and not "Man". 
530 Apply transformation rules to generate equivalent sentences

531 Employing the synonyms and the transformation rules, enables us to generate a huge number of

532 sentences that are equivalent, in meaning and label, to the original input sentence. Every

533 extracted synonym, using Arabic WordNet, creates a new sentence from the input sentence.

534 Subsequently, these sentences are processed by the transformation module which selectively

535 applies the proper transformation rules and generates even more sentences with the same

536 meaning and label to the original sentence. Meaning, here, is defined in the loose sense of being

537 suitable for sentiment analysis and is not from a linguistics perspective. From a linguistic

538 perspective, synonymous sentences represent close meanings but not exactly the same. As an

539 example, one can generate 47 sentences from the simple verbal sentence ("أكل الولد التفاحة") (The

540 boy ate the apple) using only the synonyms and transformation rules (i.e. without using the

541 negation module which would generate even more sentences). Table 6 shows a sample of 9

542 sentences generated from the example sentence (The boy ate the apple).

543

544 Generate Parse Trees

545 This module is responsible for generating parse trees for the original input sentence; and the

546 generated sentences using Stanford Arabic parser tagset. With parse trees, it becomes easier to

547 apply the suitable transformation rules to a given sentence, and it also facilitates the infusion of

548 negation particles into sentences as described in the next section. Part (A) of Figure 2 depicts the

549 parse tree of the sentence (أكل الولد التفاحة) (ate the boy the apple), while part (B) of Figure 2 shows

550 the parse tree of the sentence (الولد أكل التفاحة) (the boy ate the apple). These two parse trees are

551 equivalent.

552

553

\section{Negation}

554 Negating a sentence in Arabic means inserting one of the negation particles used in Arabic into

555 an affirmative sentence. Every negation particle, in Arabic, has its own rules in terms of the type

556 of verbs or nouns it affects and in terms of the position in the sentence in which it is inserted.

557 Negating a sentence will result in a new sentence that has an opposite meaning to the original input sentence. The label of the input sentence is also flipped from positive to negative. In addressing the negation problem, we adopted the Negation-aware Framework presented by Duwairi and Alshboul [70], where the authors explore the effects of Arabic morphology on

561

562

563

564

565

566

567

568 sentiment analysis. The study focused on five negations particles (لئ لن، لا، ما، ليس) that have been grouped into two categories based on their effect on the word as shown in Table 7.

After defining the negation rules, the system is able to negate a set of sentences and generate all possible variations of these sentences as a result of inserting negation particles regardless if the sentences are nominal or verbal sentences. Table 8 shows an example of the output generated after applying negation to the positive verbal sentence (أعجب الولد الطعام) which means (The boy likes the food). As it can be seen from Table 8, this one sentence generates 10 sentences with 
569 opposite labels (i.e. the input sentence shows positive sentiment towards food while the 10

570 generated sentences convey negative sentiments towards food).

571

\section{Evaluation}

573 The following subsections describe, thoroughly, three experiments that were designed to test the

574

575

576

577

578

579

580

581

582

583

584

585

586

587

588

589

590

591

592

593

594

595

596

597

598

599

600

601

602

603

604

605

606

607

accuracy of the proposed augmentation framework. Firstly, an assessment for the impact of the proposed framework on sentiment analysis was made. Secondly, we tested the correctness of each transformation rule. Finally, the accuracy of the Negation module was tested and formulated.

\section{Experiment1: classification of sentiment towards products}

The aim of this experiment is to classify product reviews into positive, negative or neutral reviews. The focus of this experiment is not the classifier, but to assess the resulting accuracy of using the proposed framework when enlarging the size of the dataset. To perform the first experiment, we used a subset of a public dataset of product reviews [71] which contains 300 reviews written in Arabic collected from souq.com. The data was annotated with three labels (1: positive 0 : Neutral, -1 : negative). In this experiment, and before performing any changes on the original data, the data was tested using several supervised classifiers (Naive Bayes, K-nearest neighbor, and Support vector machine). The data was divided into $70 \%$ for training and $30 \%$ for testing. All the classifiers used word embedding that is generated using AraVec with a dimension equals to 300 [72]. After the training process for each classifier, the testing phase for each classifier's performance and ability to classify the testing data was performed. Accuracy was used to assess the performance of each classifier. Accuracy is calculated by dividing the number of correctly classified reviews by the number of all reviews. The reported accuracy was equal to $54.18 \%$ using the SVM classifier, $49.99 \%$ using the Naïve Bayes classifier, and $52.17 \%$ using the K-nearest neighbor classifier. Next, the data was fed into the augmentation tool where the size of the data was increased by almost 10 times. The generated dataset was tested using the same classifiers. In comparison with the previous results, the accuracy was increased by $42 \%$ on average. In details, the accuracy rates obtained by each classifier, using the augmented dataset, were $97 \%$ using the SVM, $87 \%$ using the NB and $91.66 \%$ using the K-nearest neighbor as illustrated in Figure 3. This improvement was expected - as increasing the dataset size will subsequently improve the training process which leads to improving the overall performance of the classifier.

\section{Experiment 2: Testing the efficiency of each transformation rule}

The aim of this experiment was to test the accuracy of each transformation rule independently. To achieve this goal, it was preferable to design a small artificial dataset, which consists of 40 statements with positive sentiment, 32 statements with negative sentiment, and 27 neutral statements. A total of 99 sentences were carefully designed to align with the 23 transformation

Peer) Comput. Sci. reviewing PDF | (CS-2020:10:54813:1:2:NEW 27 Feb 2021) 
608 rules. Each sentence was processed by the augmentation tool, and thus several sentences were

609

610

611

612

613

614

615

616

617

618

619

620

621

622

623

624

625

626

627

628

629

630

631

632

633

634

635

636

637

638

639

640

641

642

643

644

645

646

647

generated for each input sentence. The generated sentences were manually inspected to test their validity. Rule accuracy is a measure that evaluates the ability of a given rule to generate correct and meaningful sentences. Rule accuracy is calculated by dividing the number of correct sentences generated by a given rule by the number of all sentences generated by that rule. "A correct sentence" means a grammatically correct and meaningful sentence. Table 9 shows the accuracy that was obtained for each rule. As can be seen from the table, all of the rules secured high accuracies. This means that the rules are capable of generating correct sentences. When examining the sources of error, we discovered that it was caused by improper synonymous words generated by the Arabic WordNet. It is important to note here that Arabic WordNet covers only $9.7 \%$ of the Arabic lexicon or vocabulary.

\section{Experiment 3: The efficiency of negation rules}

The goal of the third experiment is to assess the capability of the Negation module in order to generate correct sentences. A small artificial dataset which consists of 26 positive sentences and 24 negative sentences was created for this purpose. It should be mentioned here that the Negation module is responsible for inserting proper negation particles into the input sentences. Negation flips the polarity of the input sentence. This means that positive sentences will become negative and vice versa. All the resulted sentences from the Negation module are correct with their respective labels properly flipped.

\section{Conclusion}

In this study, a novel data augmentation framework for Arabic textual datasets for sentiment analysis was presented. In total, 23 transformation rules were designed to generate new sentences from the input ones. These rules were designed after carefully inspecting Arabic morphology and syntax. To increase the number of generated sentences for every rule, Arabic WordNet was used to swap the words with their respective synonyms. These rules preserve the labels of the input sentences. This means that if the input sentence has a positive label then the generated sentences also have positive labels. By the same token, if the label of the input sentence is negative, the labels of the generated sentences are also negative. The same is true for the neutral label. A Negation module was also designed to insert negation particles into Arabic sentences. This module inverts or flips the labels of the generated sentences, as this is the effect of negation particles on the polarity of statements. Experimentally, we tested the proposed framework by conducting three experiments. The first experiment has demonstrated the effect of increasing the dataset size, using the augmentation tool, on classification. As expected, the accuracy improved in all the classifiers. This indicates that the quality of the generated sentences was high. The second experiment was designed to test the accuracy of each transformation rule. An artificial dataset was designed for this purpose. All rules scored extremely high accuracies. The third and last experiment used an artificial dataset to assess the quality of the generated sentences from the

Peer] Comput. Sci. reviewing PDF | (CS-2020:10:54813:1:2:NEW 27 Feb 2021) 
648 Negation module. The experiment reveals that all generated sentences were correct with proper

649

650

651

652

653

654

655

656

657

658

659

660

661

662

663

664

665

666

667

668

669

670

671

672

673

674

675

676

677

678

679

680

681

682

683

684

685

686

687

688

689

690

associated labels.

\section{Acknowledgement}

This work was supported by Jordan University of Science and Technology, Jordan. Grant No. (20160150).

\section{References}

[1] Stefan Weninger, Geoffrey Khan, Michael P. Streck, Janet C. E.Watson (ed). Semitic languages: an international handbook, Walter de Gruyter gmbh \& Co. Berlin/Boston, 2011.

[2] Ibrahim Al-Huri. Arabic Language: Historic and Sociolinguistic Characteristics. 2015. English Literature and Language Review, Vol 1, Issue 4, pages 28-36.

[3] Summary by language size. Ethnologue. https://www.ethnologue.com/statistics/summarylanguage-size-19. Last retrieved 30-10-2020.

[4] Documentation for ISO 639 identifier: ara. https://iso639-3.sil.org/code/ara. Last retrieved 30-10-2020.

[5] Korayem Mohammed, David Crandall, and Muhammad Abdul-Mageed. Subjectivity and sentiment analysis of Arabic: A survey. International conference on advanced machine learning technologies and applications. Springer, Berlin, Heidelberg, 2012.

[6] Mona Diab, Musa Alkhalifa, Sabri Elkateb, Christiane Fellbaum, Aous Mansouri, Martha Palmer. Semeval-2007 task 18: Arabic semantic labeling. Proceedings of the Fourth International Workshop on Semantic Evaluations (semeval-2007). 2007.

[7] Diab, Mona, Kadri Hacioglu, and Daniel Jurafsky. Automatic tagging of Arabic text: From raw text to base phrase chunks. Proceedings of HLT-NAACL 2004: Short papers. Association for Computational Linguistics, 2004.

[8] Green, Spence, and Christopher D. Manning. Better Arabic parsing: Baselines, evaluations, and analysis. Proceedings of the 23rd International Conference on Computational Linguistics. Association for Computational Linguistics, 2010.

[9] Stanford Arabic parser tagset | Sketch Engine, Sketchengine.co.uk, 2018. [Online]. Available: https://www.sketchengine.co.uk/stanford-arabic-parser-tagset/. Last retrieved 3010-2020.

[10] Sarah Al-Humoud, Mawaheb Altuwaijri, Tarfa AlBuhairi, Wejdan Alohaideb. Survey on Arabic sentiment analysis in Twitter. International Science Index 9.1 (2015): 364-368.

[11] Ahmed Oussous, Fatima-Zahra Benjelloun, Ayoub Ait Lahcen, Samir Belfkih. 2020. ASA: A framework for Arabic sentiment analysis. Journal of Information Science, vol. 46, Issue 4, pages 544-559, https://doi.org/10.1177/0165551519849516.

[12] Abubakr H. Ombabi, Wael Ouarda, Adel M. Alimi. 2020. Deep learning CNN-LSTM framework for Arabic sentiment analysis using textual information shared in social networks. Social Network Analysis and Mining, Volume 10, issue 53, (2020). https://doi.org/10.1007/s13278-020-00668-1

[13] Rehab Duwairi, Mosab Alfaqeh, Sentiment Analysis for Arabizi Text. The 7th International Conference on Information and Communication Systems (ICICS), Irbid, Jordan, 5-7 April, 2016. 
691

692

693

694

695

696

697

698

699

700

701

702

703

704

705

706

707

708

709

710

711

712

713

714

715

716

717

718

719

720

721

722

723

724

725

726

727

728

729

730

731

732

733

734

735

[14] Ali Ahmed, Stephan Vogel, and Steve Renals. Speech recognition challenge in the wild: Arabic MGB-3. 2017 IEEE Automatic Speech Recognition and Understanding Workshop (ASRU). IEEE, 2017.

[15] Eiman Alsharhan, and Allan Ramsay. Improved Arabic speech recognition system through the automatic generation of fine-grained phonetic transcriptions. Information Processing \& Management. Vol. 56, Issue 2, 2019, pages: 343 - 353.

[16] Michel Galley, Spence Green, Daniel Cer, Pi-Chuan Chang, Christopher D. Manning (2009). Stanford University's Arabic-to-English Statistical Machine Translation System for the 2009 NIST Evaluation. The 2009 NIST Open Machine Translation Evaluation Meeting. Ottawa, Canada.

[17] Ruqyya. Al-Ibrahim, Rehab Duwairi. Neural Machine Translation from Jordanian Dialect to Modern Standard Arabic, 11th International Conference on Information and Communication Systems, Irbid, 2020.

[18] Bara Bataineh, Rehab Duwairi and Malak Abdullah, ArDep: An Arabic Lexicon for Detecting Depression, The 3rd International Conference on Advances in Artificial Intelligence (ICAAI 2019), Istanbul, Turkey, 26-28 Oct. 2019.

[19] Sosuke Kobayashi. Contextual Augmentation: Data Augmentation by Words with Paradigmatic Relations. Proceedings of the 2018 Conference of the North American Chapter of the Association for Computational Linguistics: Human Language.

[20] Oumaima Oueslati, Erik Cambria, Moez Ben HajHmida, Habib Ounelli, A review of sentiment analysis research in Arabic language, Future Generation Computer Systems 112 (2020), pp. 408-430.

[21] Mahmoud Al-Ayyoub, Abed Allah Khamaiseha, Yaser Jararweha, Mohammed N. AlKabib, A comprehensive survey of Arabic sentiment analysis. Information Processing and Management, 56 (2019), pp. 320 - 342.

[22] Yang Liu, Xiangji Huang, Aijun An, Xiaohui Yu, ARSA: a sentiment-aware model for predicting sales performance using blogs, in: Proceedings of the 30th Annual International ACM SIGIR Conference on Research and Development in Information Retrieval, ACM, 2007, pp. 607-614.

[23] Andrea Ceron, Luigi Curini, Stefano M. Iacus, Using sentiment analysis to monitor electoral campaigns: Method matters - evidence from the United States and Italy, Soc. Sci. Comput. Rev. 33 (1) (2015) 3-20.

[24] Monireh Ebrahimi, Amir Hossein Yazdavar, Amit Sheth. Challenges of Sentiment Analysis for Dynamic Events. IEEE Intelligent Systems, 2017, vol. 23, Issue 5.

[25] Shanshan Gao, Jinxing Hao, Yu Fu, The application and comparison of web services for sentiment analysis in tourism, in: Service Systems and Service Management (ICSSSM), 2015 12th International Conference on, IEEE, 2015, pp. 1- 6.

[26] Erik Cambria, Marco Grassi, Amir Hussain, Catherine Havasi, Sentic computing for social media marketing, Multimedia Tools and Applications, 59 (2) (2012) 557-577.

[27] Rehab Duwairi, Nizar Ahmed, Saleh Al-Rifai. Detecting sentiment embedded in Arabic social media - A lexicon-based approach. Journal of Intelligent \& Fuzzy Systems, 2015, 29(1), pp. 107-117.

[28] Rehab Duwairi, \& Mahmoud El-Orfali. A study of the effects of preprocessing strategies on sentiment analysis for Arabic text. Journal of Information Science, 2014, 40(4), pp. 501-513. 
736

737

738

739

740

741

742

743

744

745

746

747

748

749

750

751

752

753

754

755

756

757

758

759

760

761

762

763

764

765

766

767

768

769

770

771

772

773

774

775

776

777

778

779

780

781

[29] Siaw Ling Lo, Erik Cambria, Raymond Chiong, David Cornforth. Multilingual sentiment analysis: from formal to informal and scarce resource languages. Artificial Intelligence Review, (2017), 48:499-527.

[30] David Vilares, Haiyun Peng, Ranjan Satapathy, Erik Cambria. BabelSenticNet: A Commonsense Reasoning Framework for Multilingual Sentiment Analysis. 2018 IEEE Symposium Series on Computational Intelligence (SSCI)

[31] Andrea Esuli, Alejandro Moreo, Fabrizio Sebastiani. Cross-Lingual Sentiment Quantification. IEEE Intelligent Systems, 2020, vol. 35, Issue 3.

[32] S. Zhai , and Z.M. Zhang. 2016. Semisupervised autoencoder for sentiment analysis. In Thirtieth AAAI Conference on Artificial Intelligence.

[33] Tang, D., B. Qin, and T. Liu. 2015. Learning semantic representations of users and products for document level sentiment classification. In Proceedings of the 53rd Annual Meeting of the Association for Computational Linguistics and the 7th International Joint Conference on Natural Language Processing, 1014-1023.

[34] Tang, D., B. Qin, and T. Liu. 2015. Document modeling with gated recurrent neural network for sentiment classification. In Proceedings of the 2015 Conference on Empirical Methods in Natural Language Processing, 1422-1432.

[35] Zhou, H., L. Chen, F. Shi, and D. Huang. 2015. Learning bilingual sentiment word embeddings for cross-language sentiment classification. In Proceedings of the 53rd Annual Meeting of the Association for Computational Linguistics and the 7th International Joint Conference on Natural Language Processing, 430-440.

[36] Le, Q., and T. Mikolov. 2014. Distributed representations of sentences and documents. In International Conference on Machine Learning, 1188-1196.

[37] Abeer Salih Hadi. Factors of Structural Ambiguity in English and Arabic: A Contrastive Study. Psychology, 2015.

[38] Daimi Kevin. Identifying syntactic ambiguities in single-parse Arabic sentence. Computers and the Humanities, 35, 3. 2001. Pages: 333-349.

[39] The Stanford Natural Language Processing Group, Nlp.stanford.edu, 2018. [Online]. Available: https://nlp.stanford.edu/software/lex-parser.shtml.

[40] G. A. Miller, R. Beckwith, C. Fellbaum, D. Gross, K.J. Miller, K. J. (1990). Introduction to WordNet: An on-line lexical database. International Journal of Lexicography, 3(4), 235-244.

[41] G. A. Miller, C. Fellbaum. 2007. WordNet then and now. Language Resources and Evaluation, 41, 209-214.

[42] G. A. Miller. 1995. WordNet: A lexical database for English. Communications of the ACM, $38,39-41$.

[43] Christiane Fellbaum, Piek Vossen. Connecting the universal to the specific: Towards the global grid. International Workshop on Intercultural Collaboration. Springer, Berlin, Heidelberg, 2007.

[44] Giannis Varelas, Epimenidis Voutsakis, Paraskevi Raftopoulou, Euripides Petrakis, Evangelos Milios. Semantic similarity methods in WordNet and their application to information retrieval on the web. Proceedings of the 7th annual ACM international workshop on Web information and data management. ACM, 2005.

[45] Piek Vossen. EuroWordNet: a multilingual database of autonomous and language specific wordnets connected via an Inter-Lingual-Index. International Journal of Lexicography, (2004),Vol.17 Issue 2, pages 161-173. https://doi.org/10.1093/ijl/17.2.161

Peer) Comput. Sci. reviewing PDF | (CS-2020:10:54813:1:2:NEW 27 Feb 2021) 
782

783

784

785

786

787

788

789

790

791

792

793

794

795

796

797

798

799

800

801

802

803

804

805

806

807

808

809

810

811

812

813

814

815

816

817

818

819

820

821

822

823

824

825

826

[46] Benoît Sagot, Darja Fišer. Extending WordNets by learning from multiple resources, HAL Archive, https://hal.archives-ouvertes.fr/hal-00655785, last accessed 12-Feb-2021.

[47] S. Elkateb, W. Black, H. Rodri'guez, M. Alkhalifa, P. Vossen, A. Pease, C. Fellbaum. (2006). Building a WordNet for Arabic. In Proceedings of the fifth international conference on language resources and evaluation (LREC 2006).

[48] Musa Alkhalifa, Horacio Rodríguez, Automatically Extending NE coverage of Arabic WordNet using Wikipedia. 3rd International Conference on Arabic Language Processing (CITALA'09), May 4-5, 2009, Rabat, Morocco.

[49] Musa Alkhalifa and Horacio Rodríguez. Automatically Extending Named Entities

Coverage of Arabic WordNet using Wikipedia. International Journal on Information and Communication Technologies, Vol. 3, No. 3, June 2010.

[50] Gilbert Badaro and Hazem Hajj, Nizar Habash. A Link Prediction Approach for Accurately Mapping a Large-scale Arabic Lexical Resource to English WordNet. ACM Trans. Asian Low-Resource Language Information Processing, Vol. 19, No. 6, Article 80. pp. 1-38, 2020.

[51] Muhammad Shoaib, M. Nadeem Yasin, Hikmat Khan, Muhammad Saeed, Malik Khiyal. 2009. Relational WordNet model for semantic search in Holy Quran. 2009 IEEE 5th International Conference on Emerging Technologies.

[52] Manal Almaayah, Majdi Sawalha, and Mohammad Abushariah. Towards an automatic extraction of synonyms for Quranic Arabic WordNet. International Journal of Speech Technology 19.2 (2016): 177-189.

[53] Mohammed Al Zamil, Qasem Al-Radaideh. Automatic extraction of ontological relations from Arabic text. Journal of King Saud University-Computer and Information Sciences 26.4 (2014): 462-472.

[54] Alex Krizhevsky, Ilya Sutskever, and Geoffrey E Hinton. 2012. Imagenet classification with deep convolutional neural networks. In Advances in neural information processing systems. 1097-1105.

[55] Christian Szegedy, Wei Liu, Yangqing Jia, Pierre Sermanet, Scott Reed, Dragomir Anguelov, Dumitru Erhan, Vincent Vanhoucke, Andrew Rabinovich. Going deeper with convolutions. Proceedings of the IEEE conference on computer vision and pattern recognition. 2015.

[56] Navdeep Jaitly, Geoffrey E. Hinton. Vocal tract length perturbation (VTLP) improves speech recognition. Proceedings of the ICML Workshop on Deep Learning for Audio, Speech and Language. Vol. 117. 2013.

[57] Tom Ko, Vijayaditya Peddinti, Daniel Povey, Sanjeev Khudanpur. Audio augmentation for speech recognition. Sixteenth Annual Conference of the International Speech Communication Association. 2015.

[58] Toan Tran, Trung Pham, Gustavo Carneiro, Lyle Palmer, and Ian Reid. 2017. A bayesian data augmentation approach for learning deep models. In Advances in Neural Information Processing Systems. 2797-2806.

[59] Suhib. Irsheidat, Rehab Duwairi, Brain Tumor Detection Using Artificial Convolutional Neural Networks. 11th International Conference on Information and Communication Systems, Irbid, 2020.

[60] Grégory Rogez and Cordelia Schmid. 2016. Mocap-guided data augmentation for 3d pose estimation in the wild. In Advances in Neural Information Processing Systems. 3108-3116. 
827

828

829

830

831

832

833

834

835

836

837

838

839

840

841

842

843

844

845

846

847

848

849

850

851

852

853

854

855

856

857

858

859

860

861

862

863

864

865

866

867

868

869

870

871

872
[61] Gil Keren, Jun Deng, Jouni Pohjalainen, and Björn W Schuller. 2016. Convolutional Neural Networks with Data Augmentation for Classifying Speakers' Native Language. In Proceedings of the 17th International Speech Communication Association INTERSPEECH. Pages 2393-2397.

[62] Panagiotis Tzirakis, George Trigeorgis, Mihalis Nicolaou, Björn W. Schuller, and Stefanos Zafeiriou. 2017. End-to-end multimodal emotion recognition using deep neural networks. IEEE Journal of Selected Topics in Signal Processing 11, 8 (2017), 1301-1309.

[63] Justin Salamon and Juan Pablo Bello. 2017. Deep convolutional neural networks and data augmentation for environmental sound classification. IEEE Signal Processing Letters 24, 3 (2017), 279-283.

[64] Xiang Zhang, Junbo Zhao, Yann LeCun. Character-level convolutional networks for text classification. Advances in Neural Information Processing Systems. 2015.

[65] Wang, W. Y. and Yang, D. (2015). That's so annoying!!!: A lexical and frame-semantic embedding based data augmentation approach to automatic categorization of annoying behaviors using petpeeve tweets. In Proceedings of the 2015 Conference on Empirical Methods in Natural Language Processing, pages 2557- 2563.

[66] Georgios Rizos, Konstantin Hemker, Björn Schuller, Augment to Prevent: Short-Text Data Augmentation in Deep Learning for Hate-Speech Classification, CIKM '19: Proceedings of the 28th ACM International Conference on Information and Knowledge Management, November 2019 Pages 991-1000, https://doi.org/10.1145/3357384.3358040.

[67] Sima Sharifirad, Borna Jafarpour, Stan Matwin. 2018. Boosting Text Classification Performance on Sexist Tweets by Text Augmentation and Text Generation using a Combination of Knowledge Graphs. 2018. Proceedings of the Second Workshop on Abusive Language Online (ALW2), pages 107-114.

[68] Oleksandr Kolomiyets, Steven Bethard, Marie-Francine Moens. Model-portability experiments for textual temporal analysis. Proceedings of the 49th Annual Meeting of the Association for Computational Linguistics: Human Language Technologies.

[69] Mueller, J. and Thyagarajan, A. (2016). Siamese recurrent architectures for learning sentence similarity. In AAAI, pages 2786-2792.

[70] Rehab Duwairi, Mohammad A. Alshboul. Negation-aware Framework for Sentiment Analysis in Arabic Reviews, The 2nd International Workshop on Social Networks Analysis, Management and Security (SNAMS - 2015), August 2015, Rome, Italy.

[71] H. ElSahar, S. El-Beltagy. (2015) Building Large Arabic Multi-domain Resources for Sentiment Analysis. In: Gelbukh A. (eds) Computational Linguistics and Intelligent Text Processing. CICLing 2015. Lecture Notes in Computer Science, vol 9042. Springer, Cham. https://doi.org/10.1007/978-3-319-18117-2 2.

[72] Abu Bakr Soliman, Kareem Eisa, and Samhaa R. El-Beltagy, “AraVec: A set of Arabic Word Embedding Models for use in Arabic NLP", in proceedings of the 3rd International Conference on Arabic Computational Linguistics (ACLing 2017), Dubai, UAE, 2017. 


\section{Table $\mathbf{1}$ (on next page)}

Statistical properties of Arabic WordNet.

Statistical properties of Arabic WordNet. 


\begin{tabular}{l|l|l} 
& Unique strings & Synsets \\
\hline Noun & 13,330 & 7961 \\
\hline Verb & 5595 & 2536 \\
\hline Named entities & 1426 & 1155 \\
\hline Broken plurals & & \\
\hline Total & 405 & 126 \\
\hline & & \\
\hline
\end{tabular}

Table 1. Statistical properties of Arabic WordNet. 


\section{Table 2 (on next page)}

Stanford Arabic parser tageset.

Stanford Arabic parser tageset. 
1

\begin{tabular}{|c|c|c|c|}
\hline Tag & Description & Tag & Description \\
\hline ADJ & Adj & NNS & Noun, plural \\
\hline $\mathrm{CC}$ & $\begin{array}{l}\text { Coordinating } \\
\text { conjunction }\end{array}$ & NOUN & Noun \\
\hline$C D$ & Cardinal number & PRP & Personal pronoun \\
\hline DT & Determiner & PRP\$ & Possessive pronoun \\
\hline DTJJ & $\begin{array}{l}\text { Adjective with the } \\
\text { determiner "Al" (ال) }\end{array}$ & PUNC & Punctuation \\
\hline DTJJR & $\begin{array}{c}\text { Adjective, comparative } \\
\text { with the determiner "Al" } \\
\text { (ال) }\end{array}$ & RB & Adverb \\
\hline DTNN & $\begin{array}{l}\text { Noun, singular or mass } \\
\text { with the determiner "Al" } \\
\text { (ال) }\end{array}$ & $\mathrm{RP}$ & Particle \\
\hline DTNNP & $\begin{array}{l}\text { Proper noun, singular } \\
\text { with the determiner "Al" } \\
\text { (ال) }\end{array}$ & $\mathrm{UH}$ & Interjection \\
\hline DTNNPS & $\begin{array}{l}\text { Proper noun, plural with } \\
\text { the determiner "Al" (ال) }\end{array}$ & VB & Verb, base form \\
\hline DTNNS & $\begin{array}{l}\text { Noun, plural with the } \\
\text { determiner "Al" (ال) }\end{array}$ & VBD & Verb, past tense \\
\hline IN & $\begin{array}{l}\text { Preposition or } \\
\text { subordinating } \\
\text { conjunction }\end{array}$ & VBG & $\begin{array}{l}\text { Verb, gerund or present } \\
\text { participle }\end{array}$ \\
\hline $\mathrm{JJ}$ & Adjective & VBN & Verb, past participle \\
\hline JJR & Adjective, comparative & VBP & $\begin{array}{l}\text { Verb, non-3rd person } \\
\text { singular present }\end{array}$ \\
\hline NN & Noun, singular or mass & $\mathrm{VN}$ & Verb, past participle \\
\hline NNP & Proper noun, singular & WP & Wh-pronoun \\
\hline NNPS & Proper noun, plural & WRB & Wh-adverb \\
\hline
\end{tabular}

Table 2. Stanford Arabic parser tageset. 


\section{Table 3(on next page)}

Transformation rules based on Arabic grammar.

Transformation rules based on Arabic grammar. 
2

\begin{tabular}{|c|c|c|}
\hline ID & Original rules & Equivalent Rules \\
\hline 1 & DTNN +ADJ & ADJ+DTNN \\
\hline 2 & $\mathrm{NN}+\mathrm{ADJ}$ & ADJ+NN \\
\hline 3 & DTNN+NN & NN+DTNN \\
\hline 4 & $\mathrm{NN}+\mathrm{NN}$ & NN + NN (swap) \\
\hline 5 & NN+DTNN & could not be changed \\
\hline 6 & DTNN+DTNN & DTNN+ DTNN (swap) \\
\hline 7 & ADJ+ADJ & ADJ +ADJ (swap) \\
\hline 8 & $P P+(N N+D T N N)$ & $\begin{array}{l}\text { Place at the beginning and } \\
\text { reverse the sentence. }\end{array}$ \\
\hline 9 & $\mathrm{PP}+(\mathrm{DTNN})$ & $\begin{array}{l}\text { Place at the beginning and } \\
\text { reverse the sentence. }\end{array}$ \\
\hline 10 & PP+ (special character VB | NN) & $\begin{array}{l}\text { Place at the beginning and } \\
\text { reverse the sentence. }\end{array}$ \\
\hline 11 & $\begin{array}{l}\text { Wh-prounoun+ end of the } \\
\text { sentences }\end{array}$ & $\begin{array}{l}\text { Place at the beginning of } \\
\text { sentences }\end{array}$ \\
\hline 12 & $\begin{array}{l}\text { Special adverb + (NN | VB | } \\
\text { (special character VB | NN)) }\end{array}$ & $\begin{array}{c}\text { (NN | VB | (special character VB } \\
\mid \text { NN)) + Special adverb }\end{array}$ \\
\hline 13 & Pronoun+ (NN |VB | ADJ) & (NN |VB | ADJ) + Pronoun \\
\hline 14 & $(\mathrm{NN} \mid \mathrm{DTNN})+\mathrm{VB}$ & $\mathrm{VB}+(\mathrm{NN} \mid \mathrm{DTNN})$ \\
\hline 15 & $\mathrm{NN}+($ Special-character +VB) & (special-character) $+\mathrm{NN}$ \\
\hline 16 & $\mathrm{VB}+(\mathrm{NN} \mid \mathrm{DTNN})$ & $(\mathrm{NN}+\mathrm{DTNN})+\mathrm{VB}$ \\
\hline 17 & $\begin{array}{c}\text { VB+(Special- } \\
\text { character+(NN|DTNN)) }\end{array}$ & $\begin{array}{c}\text { (Special-character+(NN|DTNN)) } \\
+ \text { VB }\end{array}$ \\
\hline 18 & $\begin{array}{c}\text { (Special-character +VB })+ \\
(\text { Special-character+(NN|DTNN }))\end{array}$ & $\begin{array}{c}\text { (Special-character+(NN|DTNN)) } \\
+(\text { Special-character +VB })\end{array}$ \\
\hline 19 & $\begin{array}{c}\text { (Special-character +VB }) \\
+(\text { NN|DTNN })\end{array}$ & $\begin{array}{c}(\mathrm{NN} \mid \mathrm{DTNN})+(\text { Special-character } \\
+ \text { +VB })\end{array}$ \\
\hline 20 & $\begin{array}{c}\text { Special-character+(NN|DTNN)) } \\
+ \text { VB }\end{array}$ & $\begin{array}{c}\text { VB+(Special- } \\
\text { character+(NN|DTNN)) }\end{array}$ \\
\hline 21 & $\begin{array}{c}\text { (Special-character+(NN|DTNN)) } \\
+(\text { Special-character +VB })\end{array}$ & $\begin{array}{c}\text { (Special-character +VB })+ \\
(\text { Special-character+(NN|DTNN) }\end{array}$ \\
\hline 22 & $\mathrm{CD}+(\mathrm{NN}|\mathrm{DTNN}| \mathrm{VB})$ & could not be changed \\
\hline 23 & $\begin{array}{c}\text { WH-Adverb } \\
+(\text { NN|VB|DTNN|(Special- } \\
\text { character+(NN|DTNN)) | } \\
(\text { Special-character +VB }))\end{array}$ & $\begin{array}{c}\text { (NN|VB|DTNN|(Special- } \\
\text { character + (NN|DTNN)) | } \\
) \text { Special-character + VB) })+ \text { WH- } \\
\text { Adverb }\end{array}$ \\
\hline
\end{tabular}




\section{Table 4(on next page)}

List of special adverbs, negation and special words

List of special adverbs, negation and special words 
1

\begin{tabular}{|c|c|c|c|c|c|}
\hline Word & Transliteration & Meaning & Word & Transliteration & Meaning \\
\hline قبل & qbl & Before & ! & Ena & that \\
\hline بعد & bEd & After & أن & Ana & that \\
\hline فوق & fwq & Above & فإن & fA'n & Then \\
\hline تحت & $\mathrm{tHt}$ & Under & لو & IW & If \\
\hline أسفل & Asfl & Down & كي & ky & So that \\
\hline أمام & A mAm & In front of & لكي & lky & in order to \\
\hline وراء & wrA' & behind & قد & qd & may \\
\hline أعلى & AEIY & Top & لكن & Ikn & But \\
\hline وسط & wsT & Center & ل5 & $\mathrm{Im}$ & did not \\
\hline ع ع & End & At & ما & $\mathrm{mA}$ & What \\
\hline خلف & xIf & behind & بعض & $\mathrm{bED}$ & Some \\
\hline شمال & $\$ m A l$ & north & فقط & $\mathrm{fqT}$ & Just \\
\hline جنوب & jnwb & South & ليت & lyt & wish \\
\hline شرق & $\$ r q$ & east & لعل & IEl & Might \\
\hline غرب & grb & West & ذي & ${ }^{*} \mathrm{y}$ & The \\
\hline يمين & ymyn & right & ليس - ل ل & lys & Not \\
\hline بسـار & ysAr & left & إذا & $E * A$ & if \\
\hline كل & $\mathrm{KL}$ & Each & & & \\
\hline
\end{tabular}

2

Table 4. List of special adverbs, negation and special words 


\section{Table 5 (on next page)}

Examples of synsets for "Man - رجل" using Arabic WordNet.

Examples of synsets for “Man - رجل" using Arabic WordNet. 
1

\begin{tabular}{|c|c|c|}
\hline Arabic & Transliteration & Meaning in English \\
\hline ذكر & ${ }^{*} \mathrm{kr}$ & Male \\
\hline عاشق & $\mathrm{EA} \$ \mathrm{q}$ & Lover \\
\hline حبيب & Hbyb & Lover \\
\hline قرين & qryn & consort \\
\hline محبوب & $\mathrm{mHbwb}$ & Lover \\
\hline زوج & zwj & Husband \\
\hline قدم & qadam & Foot \\
\hline ساق & saAq & Leg \\
\hline
\end{tabular}

Table 5. Examples of synsets for "Man - رجل using Arabic WordNet. 


\section{Table 6(on next page)}

Examples of some equivalent sentences generated from the statement (أكل الولد التفاحة)

Examples of some equivalent sentences generated from the statement (أكل الولد التفاحة) 
1

Original: أكل الولا التفاحة

\begin{tabular}{|c|c|c|}
\hline WordNet Synonymous & & Rules Possibilities \\
\hline \multirow[t]{3}{*}{ الولد : Subject } & 1 & أكل الولد التفاحة \\
\hline & 2 & الولد أكل التفاحة \\
\hline & 3 & أكل التفاحة الولد \\
\hline \multirow[t]{3}{*}{ Subject: الثناب } & 4 & أكل الثاب التفاحة \\
\hline & 5 & الثاب أكل التفاحة \\
\hline & 6 & أكل التفاحة الثـاب \\
\hline \multirow[t]{3}{*}{ Subject: الفتى } & 7 & أكل الفنى التفاحة \\
\hline & 8 & الفنى أكل التفاحة \\
\hline & 9 & أكل التفاحة الفنى \\
\hline
\end{tabular}




\section{Table 7 (on next page)}

Negation particles and their effects in Arabic [48]

Negation particles and their effects in Arabic [48] 
1

\begin{tabular}{c|c|c|l}
$\begin{array}{c}\text { Negation } \\
\text { Particle }\end{array}$ & $\begin{array}{c}\text { BuckWalter } \\
\text { Transliteration }\end{array}$ & Category & \multicolumn{1}{c}{ Effect } \\
\hline$ل$ & lam & Group A & Affects the verb after the particle \\
\hline$ل$ J & Lan & Group A & Affects the verb after the particle \\
\hline$\gamma$ & lA & Group A & Affects the verb after the particle \\
\hline m & mA & Group A & Affects the verb after the particle \\
\hline laysa & Group B & $\begin{array}{l}\text { Affects the following two nouns or affects the } \\
\text { following verb. }\end{array}$ \\
\hline & & &
\end{tabular}

Table 7. Negation particles and their effects in Arabic [48] 


\section{Table 8 (on next page)}

Examples of negated Arabic verbal sentences.

Examples of negated Arabic verbal sentences. 
1

\begin{tabular}{|c|c|}
\hline Negation Particles & Generated Sentences \\
\hline \multirow[t]{2}{*}{ 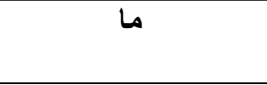 } & ما أعجب الولا الطعام \\
\hline & الطعام ما أعجب الولد \\
\hline \multirow[t]{2}{*}{ لم } & لم يعجب الولا الطعام \\
\hline & الطعام لم يعجب الولا \\
\hline \multirow[t]{2}{*}{ لن } & لن يعجب الطعام الولد \\
\hline & الطعام لن يعجب الولد \\
\hline \multirow[t]{2}{*}{ ل } & لا يعجب الطعام الولد \\
\hline & الطعام لا بعجب الولد \\
\hline \multirow[t]{2}{*}{ ل ل ليس } & ليس يعجب الولا الطعام \\
\hline & ليس الطعام يعجب الولد \\
\hline
\end{tabular}

Table 8. Examples of negated Arabic verbal sentences. 


\section{Table 9 (on next page)}

Accuracy rate per transformation rule

Accuracy rate per transformation rule 
1

\begin{tabular}{l|l|l|l|l} 
Rule 1 & Rule 2 & Rule 3 & Rule 4 & Rule 5 \\
\hline 77.5 & 89.3 & 90.07 & 94.28 & 97.53 \\
\hline Rule 6 & Rule 7 & Rule 8 & Rule 9 & Rule 10 \\
\hline 91.50 & 96.36 & 94.4 & 100 & 100 \\
\hline Rule 11 & Rule 12 & Rule 13 & Rule 14 & Rule 15 \\
\hline 98.79 & 96.96 & 100 & 100 & 100 \\
\hline Rule 16 & Rule 17 & Rule 18 & Rule 20 \\
\hline 100 & 100 & 97.5 & 100 & 100 \\
\hline Rule 21 & Rule 22 & & & \\
\hline 100 & 100 & & &
\end{tabular}

Table 9: Accuracy rate per transformation rule 


\section{Figure 1}

\section{Framework for the proposed text augmentation tool}

Framework for the proposed data augmentation tool

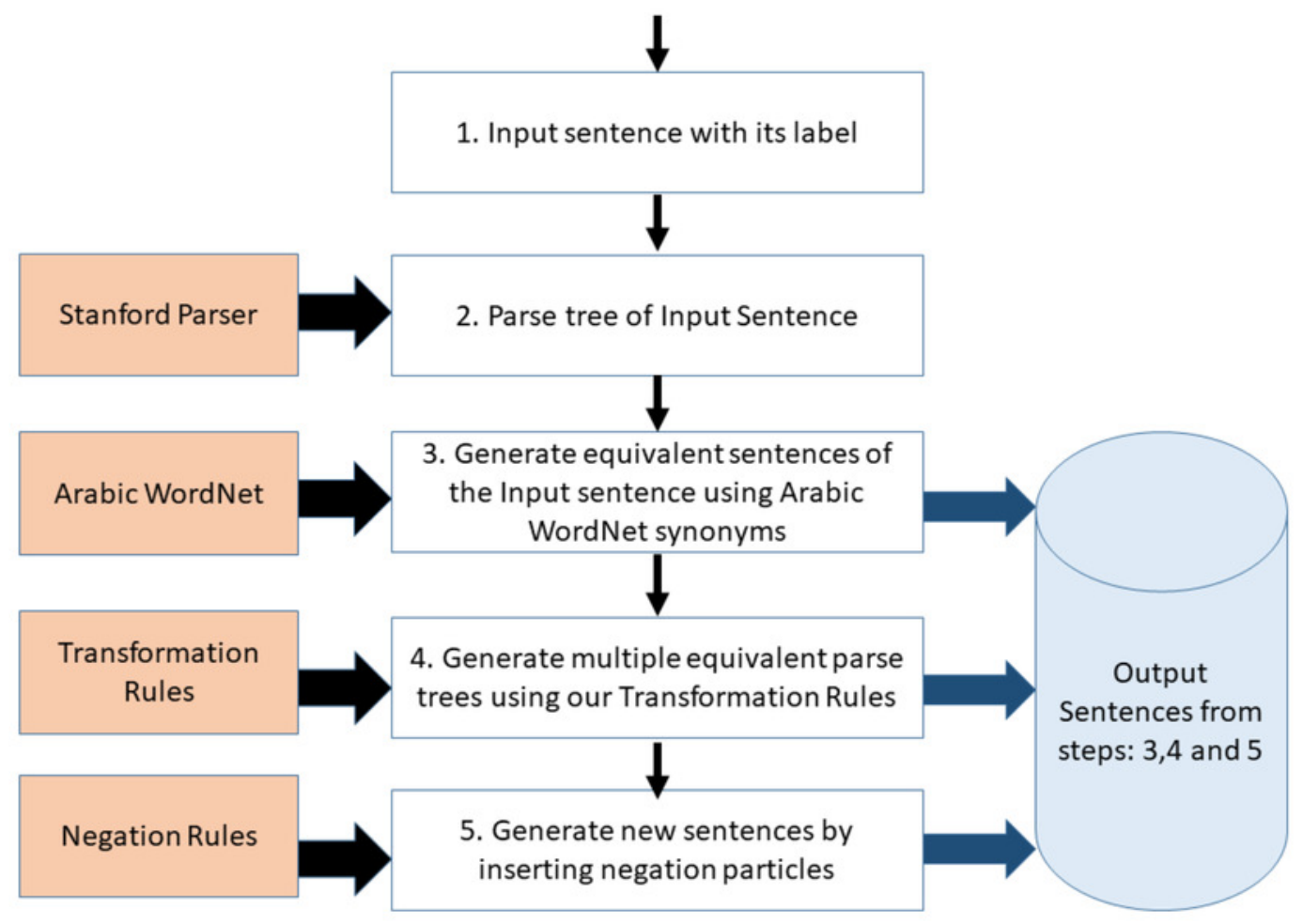


Figure 2

equivalent parsing trees.

Examples of equivalent parsing trees.

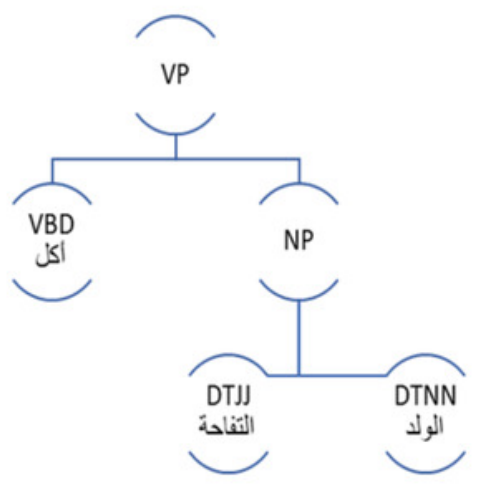

(A)

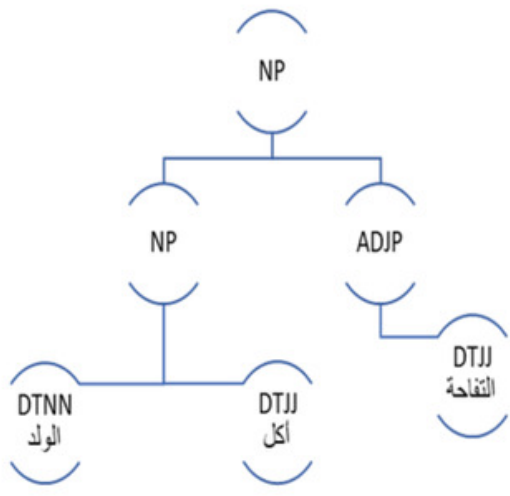

(B) 


\section{Figure 3}

Accuracy rates using the original dataset and the augmented dataset

Accuracy rates using the original dataset and the augmented dataset .

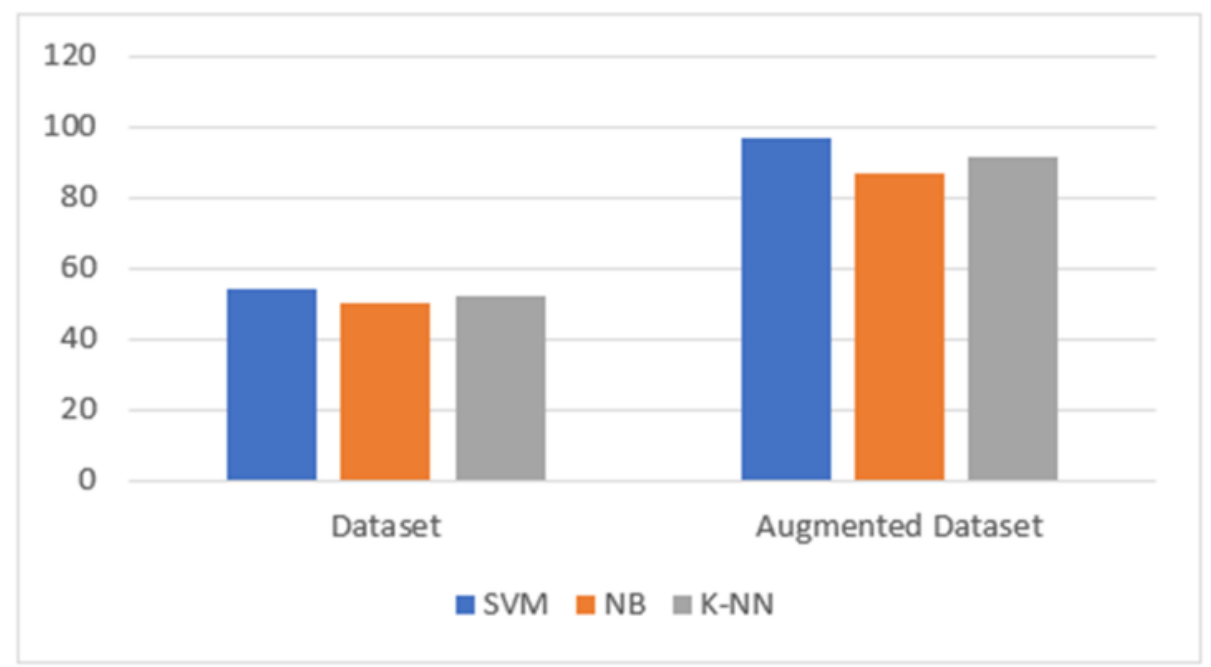

